\title{
CONVERGENCE IN TRACE IDEALS ${ }^{1}$
}

\author{
B. $\operatorname{SIMON}^{2}$
}

\begin{abstract}
We give an elementary proof of a theorem of Arazy which presents necessary and sufficient conditions on a symmetric sequence so that the associated symmetrically normed trace ideal has the property that if $A_{n} \rightarrow A$ in the weak operator topology and $\left\|A_{n}\right\| \rightarrow\|A\|$, then $\left\|A_{n}-A\right\| \rightarrow 0$.
\end{abstract}

Let $\Phi$ be a symmetric norm on finite sequences, i.e. a norm invariant under permutations and depending only on the absolute values of the coordinates. Let $s_{\Phi}$ be the associated maximal sequence space, i.e. those sequences $x=\left\{x_{n}\right\}$ with $\Phi(x) \equiv \lim _{n \rightarrow \infty} \Phi\left(\left(x_{1}, \ldots, x_{n}, 0, \ldots\right)\right)<\infty$ and let $\Phi_{\Phi}$ be the corresponding trace ideal, i.e. those compact $A$ for which $|A|$ has eigenvalues $\mu_{1}(A)>\mu_{2}(A)>\cdots>0$ with $\left\{\mu_{i}(A)\right\} \in s_{\Phi}$. We set $\Phi(A) \equiv \Phi\left(\left\{\mu_{i}(A)\right\}\right)$. To avoid certain technical questions we deal here with the maximal spaces but it is easy to extend our analysis to general symmetrically normed sequence spaces.

Here we are concerned with the following convergence property for $\mathscr{G}_{\Phi}$ :

Definition. We say that $\Phi$ has property (1) if and only if for all sequences $\left\{A_{n}\right\} \subset \mathcal{G}_{\Phi}$ and $A \in \mathcal{G}_{\Phi}$, we have that if $A_{n} \rightarrow A$ in the weak operator topology and if $\Phi\left(A_{n}\right) \rightarrow \Phi(A)$, then $\Phi\left(A-A_{n}\right) \rightarrow 0$.

For $\Phi=9_{p}, 1<p<\infty$, one can conclude that $\Phi$ has property (1) from the known uniform convexity of $g_{p}[6]$ and the elementary fact that for sequences in $g_{p}$, weak operator convergence is equivalent to weak Banach space convergence. For $\Phi=g_{p, w}, 1<p<\infty$, with the Calderon norm

$$
\Phi(x)=\sup \left\{k^{-1+p^{-1}}\left[\left|x_{j_{1}}\right|+\cdots+\left|x_{j_{k}}\right|\right] \mid j_{1}, \ldots, j_{k} \text { district }\right\}
$$

it is easy to see that property (1) fails. For $\Phi=G_{1}$ Grümm [5] proved the weaker result than property (1) where weak operator convergence of $A_{n}$ to $A$ is replaced by strong operator convergence of $A_{n}$ and $A_{n}^{*}$ to $A$ and $A^{*}$ respectively. In [7], we conjectured that property (1) holds for $G_{1}$. Independently, Arazy found an elementary proof for $G_{1}$ which he did not publish until recently as an appendix to [3].

Recently, Arazy began a progam of relating general properties of $g_{\Phi}$ to the corresponding properties of $s_{\Phi}$. In response to [2], we suggested that property (1) might be equivalent to

Definition. We say that $\Phi$ has property (2) if and only if for all sequences $\left\{x^{(k)}\right\} \subset s_{\Phi}$ and $x \in s_{\Phi}$, we have that if $x_{n}^{(k)} \rightarrow x_{n}$ for each $n$ and if $\Phi\left(x^{(k)}\right) \rightarrow \Phi(x)$, then $\Phi\left(x^{(k)}-x\right) \rightarrow 0$.

Received by the editors November 13, 1980.

1980 Mathematics Subject Classification. Primary 47D25; Secondary 46B99.

${ }^{1}$ Research partially supported by NSF Grant MCS-78-01885.

${ }^{2}$ Sherman Fairchild Visiting Scholar, at the California Institute of Technology, on leave from Departments of Mathematics and Physics, Princeton University. 
Arazy then proved

THeOREM 1 ([3]). $\Phi$ has property (1) if and only if $\Phi$ has property (2).

While [3] is short, it depends upon the rather elaborate machinery of [1]. Our goal here is to give a "bare hands" proof of Theorem 1 . Since $s_{\Phi}$ can be considered in $G_{\Phi}$ as diagonal matrices in some fixed basis, it is obvious that property (1) implies property (2), so we concentrate on the converse direction. We begin with a preliminary about sequence spaces.

DEFINITION. We say that $\Phi$ has property (3) if and only if $x, y \in s_{\Phi}$ with $0<x_{n}<y_{n}$ and $\Phi(x)=\Phi(y)$ implies that $x=y$.

LEMMA 1. If $\Phi$ has property (2), it has property (3).

Proof. (This is essentially Proposition 2 of [3] but we give an independent proof.) Suppose that property (3) fails and pick $x \neq y$ with $\Phi(x)=\Phi(y), 0<x_{n}<$ $y_{n}$. Since $\Phi(z)$ is monotone in $\left|z_{i}\right|$, we can increase some $x_{i}$ and suppose without loss that $x_{j}=y_{j}$ for all $j$ but one and by symmetry we may suppose that it is for $j=1$ that $x_{j} \neq y_{j}$. Since $y_{1}>0$, we can suppose that $y_{1}=1$. Let $f(\lambda)=$ $\Phi\left(\left(\lambda, y_{2}, y_{3}, \ldots\right)\right)$. By hypothesis, $f$ is constant on the nonempty interval $\left(x_{1}, 1\right)$ and obviously $f$ is convex in $\lambda$ and obeys $f(-\lambda)=f(\lambda)$. It follows that $f$ is constant on $(-1,1)$ so

$$
\Phi\left(\left(0, y_{2}, \ldots, y_{k}, \ldots\right)\right)=\Phi\left(\left(1, y_{2}, \ldots\right)\right)
$$

Now let

$$
x^{(k)}=\left(y_{2}, \ldots, y_{k}, 1, y_{k+1}, \ldots\right), \quad x^{(\infty)}=\left(y_{2}, \ldots, y_{k}, \ldots\right) .
$$

Then clearly $x_{n}^{(k)} \rightarrow x_{n}^{(\infty)}$ for all $n$ and by symmetry and (1), $\Phi\left(x^{(k)}\right)=\Phi\left(x^{(\infty)}\right)$. But

$$
\Phi\left(x^{(\infty)}-x^{(k)}\right)>\Phi\left(\left(1-y_{k+1}, 0,0, \ldots\right)\right)
$$

does not converge to zero so condition (2) fails.

REMARK. It is evident that condition (3) fails for $\mathscr{G}_{p, w}$ in the Calderon norm which is probably the easiest way to see that conditions (1) and (2) fail.

We also need several properties of $g_{\Phi}$ as preliminaries.

LEMMA 2. Let $\Phi$ be arbitrary. Then

(a) if $P$ is an orthogonal projection and $Q=1-P$, and if $A \in G_{\Phi}$, then $P A P+$ $Q A Q \in \mathcal{G}_{\Phi}$ and

$$
\Phi(P A P+Q A Q)<\Phi(A) .
$$

(b) If $A^{*} A$ and $B^{*} B$ lie in $\mathscr{G}_{\Phi}$, so does $A^{*} B$ and

$$
\Phi\left(A^{*} B\right)<\Phi\left(A^{*} A\right)^{1 / 2} \Phi\left(B^{*} B\right)^{1 / 2} .
$$

(c) If $A_{n}^{(j)} \rightarrow A^{(j)}, j=1, \ldots, k$, weakly with $A_{n}^{(j)}, A^{(j)} \in \mathscr{G}_{\Phi}$ then we can find an increasing sequence of finite rank projections $P_{n}$ with $s-\lim P_{n}=1$ and

$$
\Phi\left(P_{n} A_{n}^{(j)} P_{n}-A^{(j)}\right) \rightarrow 0, \quad j=1, \ldots, k
$$


Proof. (a) This is a result of Gohberg and Krein [4]; here is an elegant "folklore" proof which we learned from Arazy: Let $U$ be the unitary operator $P-Q$. Then $P A P+Q A Q=\frac{1}{2}\left(A+U A U^{-1}\right)$ so (2) follows from $\Phi\left(U A U^{-1}\right)=$ $\Phi(A)$.

(b) This is trivial if $A$ or $B$ is zero. So, without loss, we can suppose that $\Phi\left(A^{*} A\right)=\Phi\left(B^{*} B\right)=1$. Since

$$
\mu_{n}\left(A^{*}\right) \mu_{n}(B) \leqslant \frac{1}{2}\left[\mu_{n}\left(A^{*}\right)^{2}+\mu_{n}(B)^{2}\right]=\frac{1}{2}\left[\mu_{n}\left(A^{*} A\right)+\mu_{n}\left(B^{*} B\right)\right]
$$

we have using Theorem 2.8 of [7]

$$
\Phi\left(A^{*} B\right) \equiv \Phi\left(\mu_{n}\left(A^{*} B\right)\right) \leqslant \Phi\left(\mu_{n}\left(A^{*}\right) \mu_{\mu}(B)\right) \leqslant \frac{1}{2}\left[\Phi\left(A^{*} A\right)+\Phi\left(B^{*} B\right)\right] .
$$

(c) We consider the case $k=1$. The general case is similar. Let $R_{n}$ be any sequence of increasing, finite rank, projections with $s-\lim R_{n}=1$ and let $Q_{n}=1-$ $R_{n}$. Then $\Phi\left(B_{n}\right) \equiv \Phi\left(R_{n} A Q_{n}+Q_{n} A R_{n}+Q_{n} A Q_{n}\right) \rightarrow 0$ so pick $n_{1}(j)$ so with $\Phi\left(B_{n}\right)$ $<1 / 2^{j}$ for $n>n_{1}(j)$. Now pick $n_{2}(j)$ inductively so that $n_{2}(j)>n_{2}(j-1)$ and

$$
\Phi\left(R_{n_{1}(j)}\left(A-A_{n}\right) R_{n_{1}(j)}\right) \leqslant 1 / 2^{j}
$$

for $n \geq n_{2}(j)$. This may be proved by the supposed weak convergence. Now let

$$
P_{n}=R_{n_{1}(j)} \text { if } n_{2}(j)<n<n_{2}(j+1)
$$

and note that

$$
\Phi\left(P_{n} A_{n} P_{n}-A\right)<2 / 2^{j}
$$

if $n>n_{2}(j)$.

The following result about property (3) is of some independent interest.

THeOREM 2. Let $\Phi$ obey property (3). Suppose that $A_{n}, A \in \mathscr{I}_{\Phi}, \Phi\left(A_{n}\right) \rightarrow \Phi(A)$ and $A_{n} \rightarrow A$ in the weak operator topology. Then $A_{n} \rightarrow A$ in the strong operator topology.

REMARK. This result and Grümm's theorem for $G_{1}$ imply property (1) for $q_{1}$.

Proof. Let $\mu_{n}(A)$ be the singular values of $A$ and let $\varphi_{n}$ be the orthonormal set of eigenvectors of $|A|$ with $|A| \varphi_{m}=\mu_{m} \varphi_{m}$ and let $\psi_{m}$ be an orthonormal basis for $(\operatorname{Ran}|A|)^{\perp}$ so that $\left\{\varphi_{m}\right\} \cup\left\{\psi_{m}\right\}$ is orthonormal basis. Since $\|B\|<c \Phi(B)$, the $\left\|A_{n}\right\|$ are uniformly bounded, so it suffices to show that $\lim _{n \rightarrow \infty}\left\|\left(A_{n}-A\right) \eta\right\|=0$ for each $\eta \in\left\{\varphi_{m}\right\} \cup\left\{\psi_{m}\right\}$. Because of the weak convergence, it suffices to prove that $\alpha \equiv \varlimsup \lim \left\|A_{n} \eta\right\| \leqslant\|A \eta\|$. We consider the case $\eta=\varphi_{2}$. The case for other $\varphi_{n}$ and for the $\psi_{n}$ is similar. Suppose we prove that for each $l$,

$$
\Phi\left(\left(\mu_{1}, \alpha, \mu_{3}, \mu_{4}, \ldots, \mu_{l}, 0,0, \ldots\right)\right)<\Phi\left(\mu_{i}\right) .
$$

Then, by property (3), $\alpha \leqslant \mu_{2}$ and we are done.

Let $\gamma_{i}=\mu_{i}^{-1} A \varphi_{i}$, so the $\gamma_{i}$ are an orthonormal set. Let $P_{l}$ be the projection onto the orthogonal complement of $\gamma_{1}, \gamma_{3}, \gamma_{4}, \ldots, \gamma_{l}$. Let $\eta_{1}=\gamma_{1}, \eta_{3}=\gamma_{3}, \ldots, \eta_{l}=\gamma_{l}$ and define

$$
\eta_{2}^{(n)}=P_{l} A_{n} \varphi_{2} /\left\|P_{l} A_{n} \varphi_{2}\right\|
$$

(by the weak convergence $\left(1-P_{l}\right) A_{n} \varphi_{2} \rightarrow 0$ so unless $\alpha=0$, in which case the result is trivial, there is a subsequence $n_{i}$ with $\left\|P_{l} A_{n_{i}} \varphi_{2}\right\| \neq 0$ for all $i$ ). Since $\left\{\eta_{i}\right\}_{i=1}^{l}$ 
and $\left\{\varphi_{i}\right\}_{i=1}^{l}$ are orthonormal sets, we have that (see Proposition 2.6 of [7])

$$
\Phi\left(\left(\eta_{1}, A_{n} \varphi_{1}\right),\left(\eta_{2}^{(n)}, A_{n}, \varphi_{2}\right), \ldots\right)<\Phi\left(A_{n}\right) .
$$

But $\left(\eta_{k}, A_{n} \varphi_{k}\right) \rightarrow \mu_{k}$ for $k \neq 2$ and to $\alpha$ for $k=2$ and thus since we are dealing with finite sequences, the left side of (5) converges to the left side of (4). By hypothesis, the right side converges.

Corollary 1. If the hypotheses of Theorem 2 hold, then $\left|A_{n}\right| \rightarrow|A|,\left|A_{n}^{*}\right| \rightarrow\left|A_{n}^{*}\right|$ both strongly.

Proof. Since $\Phi(B)=\Phi\left(B^{*}\right)$ and weak convergence of $A_{n}$ to $A$ implies weak convergence of $A_{n}^{*}$ to $A^{*}$, we have strong convergences of $A_{n}^{*}$ and $A_{n}$ to $A^{*}$ and $A$ respectively. Because of the uniform bound on $\left\|A_{n}\right\|, A_{n}^{*} A_{n} \rightarrow A^{*} A$ strongly, so that $\left|A_{n}\right| \rightarrow|A|$ by the strong continuity of the map $B \mapsto \sqrt{B}$ on positive operators.

LEMMA 3. Let $\Phi$ have property (2). Let $P_{n}$ be a sequence of finite rank projections so that $\Phi\left(P_{n} A_{n} P_{n}-A\right) \rightarrow 0, P_{n} \rightarrow 1$, strongly, and suppose that $\Phi\left(A_{n}\right) \rightarrow \Phi(A)$. Let $Q_{n}=1-P_{n}$. Then $\Phi\left(Q_{n} A_{n} Q_{n}\right) \rightarrow 0$.

Proof. Let $B_{n}=Q_{n} A_{n} Q_{n}+P_{n} A_{n} P_{n}$. Using (2)

$$
\Phi\left(P_{n} A_{n} P_{n}\right)<\Phi\left(B_{n}\right)<\Phi\left(A_{n}\right)
$$

so

$$
\Phi\left(B_{n}\right) \rightarrow \Phi(A) .
$$

Let $x^{(n)}$ be the sequence obtained by first listing all the singular values of $P_{n} A_{n} P_{n}$ including enough zeros to have $\operatorname{dim} P_{n}$ entries and then all the singular values of $Q_{n} A_{n} Q_{n}$. Let $x$ be the sequence of singular values of $A$. Since

$$
\left\|P_{n} A_{n} P_{n}-A\right\| \rightarrow 0
$$

we have that

$$
x_{i}^{(n)} \rightarrow x_{i}
$$

for each finite $i$. By (6), $\Phi\left(x^{(n)}\right) \rightarrow \Phi(x)$ and thus, by the assumed property (2)

$$
\Phi\left(x^{(n)}-x\right) \rightarrow 0 \text {. }
$$

Let $y^{(n)}$ be the sequence of singular values of $P_{n} A_{n} P_{n}$ followed by zeros. By Theorem 1.20(a) of [7]

$$
\Phi\left(y^{(n)}-x\right)<\Phi\left(P_{n} A_{n} P_{n}-A\right) \rightarrow 0
$$

and thus, using (7)

$$
\Phi\left(Q_{n} A_{n} Q_{n}\right)=\Phi\left(y^{(n)}-x^{(n)}\right) \rightarrow 0
$$

Proof of Theorem 1. Suppose that $A_{n} \rightarrow A$ weakly and $\Phi\left(A_{n}\right) \rightarrow \Phi(A)$. Since $\Phi$ has property (2), it has property (3) by Lemma 1 and therefore by Corollary 1 $\left|A_{n}\right| \rightarrow|A|,\left|A_{n}^{*}\right| \rightarrow\left|A^{*}\right|$ strongly. Thus by Lemma 2(c), we can find an increasing sequence of projections $P_{n}$ so that $\Phi\left(P_{n} A_{n} P_{n}-A\right) \rightarrow 0, \Phi\left(P_{n}\left|A_{n}\right| P_{n}-|A|\right) \rightarrow 0$, 
$\Phi\left(P_{n}\left|A_{n}^{*}\right| P_{n}-\left|A^{*}\right|\right) \rightarrow 0$. Since $\Phi(B)=\Phi\left(B^{*}\right)=\Phi(|B|)$, we can use Lemma 3 to conclude that

$$
\Phi\left(Q_{n}\left|A_{n}\right| Q_{n}\right)+\Phi\left(Q_{n}\left|A_{n}^{*}\right| Q_{n}\right)+\Phi\left(Q_{n} A Q_{n}\right) \rightarrow 0
$$

But, by Lemma 2(b),

$$
\begin{aligned}
\Phi\left(P_{n} A_{n} Q_{n}\right) & \leqslant \Phi\left(\left|A_{n}\right| Q_{n}\right)=\Phi\left(\left|A_{n}\right|^{1 / 2}\left(\left|A_{n}\right|^{1 / 2} Q_{n}\right)\right) \\
& <\Phi\left(\left|A_{n}\right|\right)^{1 / 2} \Phi\left(Q_{n}\left|A_{n}\right| Q_{n}\right)^{1 / 2} \rightarrow 0
\end{aligned}
$$

and similarly $\Phi\left(Q_{n} A_{n} P_{n}\right)=\Phi\left(P_{n} A_{n}^{*} Q_{n}\right) \rightarrow 0$. Thus

$$
\Phi\left(A_{n}-A\right) \leqslant \Phi\left(P_{n} A_{n} P_{n}-A\right)+\Phi\left(Q_{n} A_{n} P_{n}\right)+\Phi\left(Q_{n} A_{n} Q_{n}\right)+\Phi\left(Q_{n} A_{n} P_{n}\right)
$$

converges to zero.

It is a pleasure to thank Jonathan Arazy for valuable discussions.

\section{REFERENCES}

1. J. Arazy, Basic sequences, embeddings, and the uniqueness of the symmetric structure in unitary matrix spaces, J. Funct. Anal. (to appear).

2. __ On the geometry of the unit ball of unitary matrix spaces, J. Integral Equations and Optim. Theory (to appear).

3. __ More on convergences in unitary matrix spaces, Proc. Amer. Math. Soc. 83 (1981), 44-48.

4. T. C. Gohberg and M. G. Krein, Introduction to the theory of linear nonselfadjoint operators, Transl. Math. Mono., vol. 18, Amer. Math. Soc., Providence, R.I., 1969.

5. H. Grümm, Two theorems about $C_{p}$, Rep. Math. Phys. 4 (1973), 211-215.

6. C. A. McCarthy, $c_{p}$, Israel J. Math. 5 (1967), 249-271.

7. B. Simon, Trace ideals and their applications, Cambridge Univ. Press, Cambridge, 1979.

Departments of Mathematics and Physics, Princeton University, Princeton, New Jersey 08544

Current address: Mail Code 253-37, California Institute of Technology, Pasadena, California 91125 